\title{
VALIDATION OF A UHPLC-FLD METHOD FOR THE SIMULTANEOUS QUANTIFICATION OF AFLATOXINS, OCHRATOXIN A AND ZEARALENONE IN BARLEY
}

\author{
María Ibáñez-Vea ${ }^{1}$, Laura Ana Corcuera ${ }^{2}$, Rebeca Remiro ${ }^{1}$, María Teresa \\ Murillo-Arbizu ${ }^{1}$, Elena González-Peñas ${ }^{1}$, Elena Lizarraga ${ }^{1}$ \\ ${ }^{1}$ Organic and Pharmaceutical Chemistry Department \\ ${ }^{2}$ Nutrition and Food Sciences, Physiology and Toxicology Department \\ Faculty of Pharmacy, C.I.F.A., University of Navarra \\ C/ Irunlarrea 1, 31008, Pamplona, Navarra, Spain
}

Corresponding author:

Elena González-Peñas PhD: Tel.: +34 948 425653. Fax: +34 948425652.

E-mail: mgpenas@unav.es

E-mail addresses of all coauthors: mivea@alumni.unav.es (María Ibáñez-Vea); lcorcuer@alumni.unav.es (Laura Ana Corcuera); rremiro@alumni.unav.es (Rebeca Remiro); mmurarb@alumni.unav.es (María Teresa Murillo-Arbizu PhD); elizarraga@unav.es (Elena Lizarraga PhD). 


\section{ABSTRACT}

A fast and simple UHPLC-FLD method has been developed for the simultaneous determination in barley of aflatoxins (B1, G1, B2 and G2), ochratoxin A (OTA) and zearalenone (ZEA), some of the most important mycotoxins due to their toxicity and occurrence. The procedure is based on the extraction of the six mycotoxins with a mixture of acetonitrile and water, and the purification of the extract with immunoaffinity columns before analysis. Detection of AFB1 and AFG1 is improved using a photochemical reaction. The method has been validated with satisfactory results. Limits of detection were $340 \mathrm{ng} \mathrm{kg}^{-1}$ for ZEA, $13 \mathrm{ng} \mathrm{kg}^{-1}$ for OTA and varied from 0.5 to $15 \mathrm{ng} \mathrm{kg}^{-1}$ for aflatoxins. Recovery percentages were between 78.2 and $109.2 \%$. After being validated, the method has been successfully applied to 20 barley samples cultivated in a region of northern Spain (Navarra).

\section{Keywords}

Mycotoxin, aflatoxins, ochratoxin A, zearalenone, UHPLC-FLD, barley. 


\section{Introduction}

Mycotoxins are toxic secondary metabolites produced by several fungal species growing on many agricultural commodities and processed food, either in the field or during storage (Bennett \& Klich, 2003).

The most frequent toxigenic fungi belong to the Aspergillus, Penicillium and Fusarium species with aflatoxins (AF) being produced by A. flavus, ochratoxin A (OTA) being produced by $A$. ochraceus and $P$. verrucosum and zearalenone (ZEA) being produced by F. graminearum and F. culmorum; all of which are very significant in terms of toxicity and occurrence (Pitt, 2006)

These toxins occur naturally in plant products such as cereals, nuts and dried fruit, and in their by-products as well (Bennett \& Klich, 2003, Miraglia \& Brera, 2002). Cereals represent a risk for the consumers because this product is very sensitive to mycotoxin contamination and is consumed wideworld. In fact, it is estimated that $25 \%$ of the world crop production and $20 \%$ of crop production within the European Union may be contaminated with mycotoxins (Zöllner \& Mayer-Helm, 2006), and it is considered that cereals are the main OTA source of human intake (Miraglia \& Brera, 2002).

These toxins represent a serious threat to both human and animal health. In animals, aflatoxins have demonstrated to be mutagenic, teratogenic and carcinogenic compounds, with the liver being the main target organ. OTA is a potent nephrotoxin and hepatotoxin with teratogenic, mutagenic, carcinogenic and immunosuppressive effects, even at trace levels (Zöllner \& Mayer-Helm, 2006). ZEA is a non-esteroideal estrogenic toxin which has been involved in incidents of precocious pubertal changes. Regarding human health, the International Agency for Research on Cancer (IARC) has classified aflatoxin B1 and naturally-occurring mixtures of aflatoxins as human carcinogens 
(group 1), and OTA as a possible carcinogen to humans (group 2B); ZEA was considered to be not classifiable with regard to its carcinogenicity to humans (group 3) (International Agency for Research on Cancer (IARC), 1993, International Agency for Research on Cancer (IARC), 2002).

Due to the serious effects that mycotoxins can cause in humans and animals, many countries have implemented regulations on mycotoxins in food and feed to protect their health. The European Commission has established maximum permitted levels for mycotoxins of major concern in cereals: $2 \mu \mathrm{g} \mathrm{kg}^{-1}$ for aflatoxin B1 and $4 \mu \mathrm{g} \mathrm{kg}^{-1}$ for the sum of AFB1, AFG1, AFB2 and AFG2; $5 \mu \mathrm{gg}^{-1}$ for OTA and $100 \mu \mathrm{g} \mathrm{kg}^{-1}$ for ZEA (European Commission, 2006a).

In order to assess the exposure to toxins, reliable data on the occurrence of mycotoxins in different commodities is needed (Van Egmond, Schothorst \& Jonker, 2007). The chemical diversity of mycotoxins and their varying concentration ranges in different samples is a great challenge to analytical chemists; therefore, most methods target on individual mycotoxins (Krska, Schubert-Ullrich, Molinelli, Sulyok, MacDonald \& Crews, 2008). However, it is important to consider the implications of exposure to several mycotoxins at once (Kuiper-Goodman, 1999). Knowledge regarding the possible synergistic, additive or antagonist effects of mycotoxins present in a same foodstuff on the health of animals and humans, as well as the possible relationship between the co-occurrence of mycotoxins, still remains very limited. Therefore, it is necessary to have validated analytical methods that allow simultaneous quantification of the main mycotoxins in several commodities (Anklam, Stroka \& Boenke, 2002) to be used for control, monitoring and risk assessment studies. In the past few years, efforts have been made to develop these methods (Krska, Schubert-Ullrich, Molinelli, Sulyok, 
MacDonald \& Crews, 2008). However, only a limited number of them include performance characteristics data obtained by method validation (Krska, SchubertUllrich, Molinelli, Sulyok, MacDonald \& Crews, 2008).

In the literature, methods based on HPLC analysis with either pre- or post-column derivatization for the determination of AFs, OTA or/and ZEA in cereals exit (Chan, MacDonald, Boughtflower \& Brereton, 2004, Langseth, Ellingsen, Nymoen \& Okland, 1989, Nguyen, Tozovanu, Tran \& Pfohl-Leszkowicz, 2007). These methods consist in extraction with a mixture of acetonitrile and water or chloroform and phosphoric acid, followed by purification using inmunoaffinity columns (IAC), liquid-liquid extraction (LLP) or solid phase extraction (SPE). Göbel and Lusky (2004) (Göbel \& Lusky, 2004), developed a method for the simultaneous determination of AFs, OTA and ZEA in rice and rye using IAC as purification technique, pre-column derivatization (adding TFA) and HPLC-FLD analysis; however, the validation of this procedure was not reported. Wang et al. (2008) (Wang et al., 2008) reported a method for the analysis of AFs, OTA and ZEA in air filters with IAC, photochemical derivatization (PHRED) and HPLCFLD analysis. Ofitserova, Nerkar, Pickering and Torma (2009) (Ofitserova, Nerkar, Pickering \& Torma, 2009) developed a method for the simultaneous analysis of 9 mycotoxins in corn, including AFs, OTA and ZEA, with HPLC-FLD in 60 minutes. The method requires three different clean-up procedures and two different derivatization techniques

Currently, different improvements in mycotoxin analysis have been made using new chromatographic tools. For instance, there is a strong trend towards the use of HPLCMS technique because of its universal, selective and sensitive detection (Krska \& Molinelli, 2007). However, fluorescence detection is by nature highly specific and 
sensitive; HPLC-FLD might still be superior in the area of quantitative determination, where the influence of matrix is negligible compared to possible problems that can arise with HPLC-MS quantification (Cigic \& Prosen, 2009).

Ultra-high performance liquid chromatography (UHPLC) using columns filled with particles $<2 \mu \mathrm{m}$ is a technique that shows improved sensitivity, resolution and speed compared to HPLC. This is a relatively new technique and there are very few published studies using UHPLC for multi-mycotoxins determination in food (Frenich, Martínez, Romero-González \& Aguilera-Luiz, 2009).

The aim of this paper is to present a fast, simple and validated method for the determination of aflatoxins (B1, B2, G1 and G2), ochratoxin A and zearalenone in barley. The procedure is based on the simultaneous extraction of the six mycotoxins with a mixture of acetonitrile and water, and the purification of the extract with IAC before its UHPLC-FLD analysis, using a PHRED photochemical reactor to achieve the derivatization of AFB1 and AFG1. This procedure has been successfully applied to the analysis of barley samples obtained from a region of northern Spain (Navarra).

\section{Material and methods}

\subsection{Chemical and reagents}

Aflatoxins, ochratoxin A and zearalenone dissolved in acetonitrile were purchased from Fluka (Schnelldorf, Germany) as certified reference materials. Potassium chloride, potassium phosphate dibasic and formic acid were obtained from Panreac (Barcelona, Spain) and sodium chloride, sodium phosphate dibasic and Tween 20 were obtained from Merck (Darmstadt, Germany). These reagents were of pro-analysis grade. Acetonitrile and methanol HPLC grade were supplied by Sigma-Aldrich (St. Quentin 
Fallavier, France). Millipore type I water was obtained daily from a Milli-Q waterpurifying system. Immunoaffinity columns AOZ were purchased from Vicam (Watertown, MA, USA).

Phosphate buffered saline (PBS) was prepared by dissolving potassium chloride (0.2 g), potassium phosphate dibasic $(0.2 \mathrm{~g})$, sodium phosphate dibasic (1.16 g) and sodium chloride $(8 \mathrm{~g})$ in $900 \mathrm{~mL}$ water type II. The $\mathrm{pH}$ of the solution was adjusted to 7.40 with $\mathrm{HCl}$ or $\mathrm{NaOH}$, and two drops of Tween 20 were added. Finally, the volume was adjusted to $1 \mathrm{~L}$.

\subsection{Barley samples}

Barley samples of $1 \mathrm{~kg}$ were collected during the 2007 harvest by different agricultural cooperatives and factories dedicated to the production of foodstuffs and feed in Navarra (Spain). All samples were stored at $4^{\circ} \mathrm{C}$ until their analysis.

\subsection{Standard solutions}

A stock standard solution containing $500 \mu \mathrm{g} \mathrm{L} \mathrm{L}^{-1}$ of AFB1, AFG1 and OTA, $125 \mu \mathrm{g} \mathrm{L}^{-1}$ of AFB2 and AFG2 and $20 \mathrm{mg} \mathrm{L}^{-1}$ of ZEA were prepared by diluting different standard solution volumes of each mycotoxin in a mixture of acetonitrile and methanol (50:50; $\mathrm{v} / \mathrm{v})$. Working standard solutions of 100, 10 and $1 \mu \mathrm{g} \mathrm{L} \mathrm{L}^{-1}$ of AFB1, AFG1 and OTA, 25, 2.5 and $0.25 \mu \mathrm{g} \mathrm{L}^{-1}$ of AFB2 and AFG2 and 4000, 400 and $40 \mu \mathrm{g} \mathrm{\textrm {L } ^ { - 1 }}$ of ZEA, respectively, were prepared by dilution from this stock standard solution. All prepared solutions were stored at $-20^{\circ} \mathrm{C}$ and maintained at room temperature and in darkness for 30 minutes before their use. Calibration samples were prepared by evaporating a given volume of the working standard solution under vacuum at $40^{\circ} \mathrm{C}$ in an evaporator (GeneVac). The residue was then dissolved in $150 \mu \mathrm{L}$ of a mixture (40:60) of 
acetonitrile-methanol (50:50) and water, both acidified with $0.5 \%$ formic acid. The acetonitrile extract from cereal samples were evaporated and dissolved in the same way.

\subsection{Extraction and clean up from barley samples}

The method used for mycotoxin extraction from cereal samples is based on that which was described by Göbel and Lusky (2004) (Göbel \& Lusky, 2004), with some modifications. Three hundred grams of barley were ground in a Restch ZM100 mill, using a sieve sieze of $0.75 \mathrm{~mm}$. Ten grammes of milled sample were extracted with $50 \mathrm{~mL}$ of a mixture of acetonitrile-water $(60: 40, \mathrm{v} / \mathrm{v})$ in an orbital shaker SSL1 (Stuart ${ }^{\circledR}$ ) for $30 \mathrm{~min}$. The extract was filtered by gravity and then $10 \mathrm{~mL}$ of the filtrate were mixed with $40 \mathrm{~mL}$ of PBS. The mixture was centrifuged at $6249 \mathrm{~g}$ and $4^{\circ} \mathrm{C}$ for 15 min. Fifteen millilitres of the supernatant were passed through an immunoaffinity column AOZ (Vicam), pre-conditioned with $3 \mathrm{~mL}$ of water and $10 \mathrm{~mL}$ of PBS. After the sample had passed, the column was washed with $5 \mathrm{~mL}$ of PBS and $15 \mathrm{~mL}$ of water. Finally, the column was dried with air and the mycotoxins were eluted with $3 \mathrm{~mL}$ of acetonitrile, after maintaining in contact acetonitrile and column antibodies for $5 \mathrm{~min}$. The extract was evaporated to dryness in an evaporator (GeneVac) and the residue was redissolved as was previously indicated. The sample was maintained at $4^{\circ} \mathrm{C}$ in the chromatograph tray until its analysis.

\subsection{Equipment and chromatographic conditions}

The instrument used was an Agilent Technologies 1200 rapid resolution liquid chromatographic system equipped with a fluorescence detector (G1321A model), and controlled by ChemStation B.03.02 software. Separation was achieved on an Ascentis Express (fused core technology) (Supelco) C18 column (150 mm x $2.1 \mathrm{~mm} ; 2.7 \mu \mathrm{m})$ which comprises a $1.7 \mu \mathrm{m}$ solid core and a $0.5 \mu \mathrm{m}$ porous shell. Columns with Fused- 
Core Technology provide the benefits of sub-2 $\mu \mathrm{m}$ particles, but at much lower backpressure.

A post-column photochemical derivatization was used to enhance the AFB1 and AFG1 responses, using a PHRED photochemical reactor with a mercury lamp $(\lambda=254 \mathrm{~nm})$ and a knitted reactor coil of $0.25 \mathrm{~mL}(5 \mathrm{~m} \mathrm{x} 0.25 \mathrm{~mm})$. The injection volume was $30 \mu \mathrm{L}$ and the flow rate was $0.9 \mathrm{~mL} \mathrm{~min}^{-1}$. Chromatography was performed at $60^{\circ} \mathrm{C}$ with a linear gradient of a mixture of acetonitrile and methanol (50:50; v/v) (A) and water (B), both acidified with $0.5 \%$ formic acid. The initial gradient condition was $16 \% \mathrm{~A}$ and $84 \% \mathrm{~B}$, changing linearly to $53 \% \mathrm{~A}$ and $47 \% \mathrm{~B}$ in $12 \mathrm{~min}$. Finally, the column was reequilibrated with the initial mobile phase conditions for 4 minutes. Fluorescence conditions were adjusted to obtain the better detection, recording the excitation and emission spectrums while calibration samples were analysed.

\subsection{Confirmation}

Mycotoxins confirmation was made using an Agilent Technologies 1200 liquid chromatographic system coupled to a MSD Trap XCT Plus mass spectrometry (G2447A model) equipped with an electrospray ionisation interface (ESI). The mycotoxin analysis was performed on an Ascentis Express C18 column $(150 \mathrm{~mm} \times 2.1 \mathrm{~mm} ; 2.7 \mu \mathrm{m})$ from Supelco, at $55^{\circ} \mathrm{C}$ and with a linear gradient of methanol (A) and water (B), both containing $0.1 \%$ formic acid and $5 \mathrm{mM}$ ammonium formate. The initial gradient condition was $40 \% \mathrm{~A}$ and $60 \% \mathrm{~B}$, changing linearly to $80 \% \mathrm{~A}$ and $20 \% \mathrm{~B}$ in $11 \mathrm{~min}$. The column was re-equilibrated for 4 minutes. The injection volume was $20 \mu \mathrm{L}$ and the flow rate was $0.3 \mathrm{~mL} \mathrm{~min}^{-1}$.

The mass spectrometer was operated in positive ion mode. Ionisation and spectrometric settings were optimised by infusing the separate mycotoxin solutions $\left(2-0.5 \mu \mathrm{gLL}^{-1}\right)$ 
at a flow rate of $5 \mu \mathrm{L} \min ^{-1}$ via a syringe pump. Data acquisition was performed working in multiple reaction monitoring $(\mathrm{MRM})$ mode using the $[\mathrm{M}+\mathrm{H}]^{+}$ions.

\subsection{Validation of the analytical method}

Validation of the UHPLC-FLD method was based on the following criteria: selectivity, linearity, precision (within- and between-day and analyst variability), accuracy, limit of detection and limit of quantification, recovery and robustness.

Selectivity was assured with the use of an immunoaffinity purification technique and a selective fluorescence detector. In addition, selectivity was tested by adding the mycotoxins to positive barley samples and then by observing the increase of each mycotoxin peak. Also, the retention time of mycotoxin peaks were checked in the samples in order to see if they corresponded with the retention time in the calibration samples (with a tolerance of $\pm 2.5 \%$ ). Moreover, the presence of mycotoxins was confirmed with the aid of a UHPLC-MS (ion trap) method.

In the assessment of linearity, two calibration curves were plotted in the ranges $0.6-4 \mu \mathrm{g} \mathrm{L} \mathrm{L}^{-1}$ and $4-40 \mu \mathrm{g} \mathrm{L}^{-1}$ for AFB1, AFG1 and OTA, $0.15-1 \mu \mathrm{g} \mathrm{L}^{-1}$ and $1-10 \mu \mathrm{g} \mathrm{L}^{-1}$ for AFB2 and AFG2 and $24-160 \mu \mathrm{g} \mathrm{L} \mathrm{L}^{-1}$ and $160-1600 \mu \mathrm{g} \mathrm{L}^{-1}$ for ZEA, respectively. In cereal samples, the equivalent concentration ranges were obtained using the following expression:

$$
\mathrm{C}_{\text {Barley }}=\left(\frac{\mathrm{C}_{\mathrm{STD}}}{\mathrm{CF}}\right) \cdot\left(\frac{100}{\operatorname{Rec}}\right)
$$

where, $\mathrm{C}_{\mathrm{STD}}$ is the measured vial concentration, $\mathrm{CF}$ is the concentration factor (4) and Rec is the recovery percentage for each toxin. Therefore, the ranges in barley samples were $0.15-1 \mu \mathrm{g} \mathrm{kg}^{-1}$ and $1-10 \mu \mathrm{g} \mathrm{kg}^{-1}$ for AFB1, AFG1 and OTA, $0.0375-0.25 \mu \mathrm{g} \mathrm{kg}^{-1}$ and $0.25-2.5 \mu \mathrm{g} \mathrm{kg}^{-1}$ for AFB2 and AFG2 and $6-40 \mu \mathrm{g} \mathrm{kg}^{-1}$ and 
$40-400 \mu \mathrm{g} \mathrm{kg}^{-1}$ for ZEA, respectively. Three replicates of six calibration samples were analyzed for each mycotoxin and range. Calibration curves were evaluated by the analysis of the distribution properties of the residuals: when plotting the toxin concentration versus the residual points, a random distribution without reflecting any tendency must be achieved, correlation coefficient $r>0.990$, slope of the linear calibration curve statistically different from $0(p=95 \%)$, and lastly, the intercept not statistically different from $0(\mathrm{p}=95 \%)$.

Accuracy, repeatability and intermediate precision (time factor) of the instrument were determined by analyzing calibration samples at low, medium and high levels of each calibration curve $\left(0.6,2.4,4,24,40 \mu \mathrm{g} \mathrm{L}^{-1}\right.$ for AFB1, AFG1 and OTA, 0.15, 0.6, 1, 6, $10 \mu \mathrm{g} \mathrm{L}^{-1}$ for AFB2 and AFG2 and 24, 96, 160, 960, $1600 \mu \mathrm{g} \mathrm{L}^{-1}$ for ZEA) per triplicate on one day and on three different days, respectively. The intermediate precision (analyst factor) was tested by analyzing mycotoxins standards at low, medium and high levels of the analysis range (as indicated before) by two different analysts. The accuracy has been calculated as the standard error of the mean (in \%) of the data obtained during the precision study, and the repeatability and intermediate precision were calculated as the relative standard deviation (RSD) in \%.

The limits of detection (LOD) and quantification (LOQ) were established from the results obtained in the analysis of three spiked barley samples at three different concentrations $\left(0.05,0.1,0.15 \mu \mathrm{g} \mathrm{kg}^{-1}\right.$ for AFB1, AFG1 and OTA, 0.0125, 0.025, $0.0375 \mu \mathrm{g} \mathrm{kg}^{-1}$ for AFB2 and AFG2 and 2, 4, $6 \mu \mathrm{g} \mathrm{kg}^{-1}$ for ZEA, respectively).

LOD was calculated using a method based on the calibration curve extrapolation at zero concentration. This method consists in plotting the toxin concentration versus the peak area (curve 1) and versus the standard deviation obtained for each toxin level (curve 2). In order to calculate the LOD value, the following equation was used: 


$$
L O D=\frac{y+\left(k \cdot y^{\prime}\right)}{b \cdot \sqrt{n}}
$$

with $y$ and $b$ being the values for $y$-intercept and slope, respectively, from curve $1, y$ ' being the $y$-intercept from curve 2 and $n$ being the number of replicates for each level $(\mathrm{n}=3)$. The $\mathrm{k}$ value was 3 for LOD (Asociación Española de Farmaceúticos de la Industria (Spanish Association of Industrial Pharmaceutics) (A.E.F.I), 2001).

The limit of quantification (LOQ) corresponds to the minimum concentration with adequate precision (RSD $<15 \%$ ) and recovery (between 50 and $120 \%$ for AFs and OTA; between 60 and 120\% for ZEA) values (European Commission, 2006b). The LOQ value for each mycotoxin has been included as the lowest level in the corresponding calibration curve.

Recovery of the method was tested at three concentration levels for each mycotoxin in spiked milled barley samples at $0.15,1$ and $10 \mu \mathrm{g} \mathrm{kg}^{-1}$ for AFB1, AFG1 and OTA, 0.0375, 0.25 and $2.5 \mu \mathrm{g} \mathrm{kg}^{-1}$ for AFB2 and AFG2 and 6,40 and $400 \mu \mathrm{g} \mathrm{kg}^{-1}$ for ZEA, respectively. Aliquots of ten grams of milled barley were spiked with adequate volumes of stock and working standard solutions until the desired mycotoxin concentration was reached. They were processed after 24 hours to ensure evaporation of the solvent. Recovery was determined extrapolating the absolute responses (area of toxin peak) obtained from the barley spiked samples in the calibration curve; the calculated concentration was compared with the expected concentration for a $100 \%$ recovery. The repeatability and reproducibility of this process were tested carrying out the complete sample process and recovery experiment per triplicate on one day and on three different days, respectively. All of the analytical results obtained have been corrected by recovery.

Robustness of the analytical procedure, the ability of the method to remain unaltered under small but deliberate variations in method parameters, was assessed by studying 
the influence in mycotoxin quantification of different batches of the chromatographic column, the temperature of the column compartment and the $\mathrm{pH}$ of the mobile phase. Two calibration samples (1.6 and $4 \mu \mathrm{g} \mathrm{L}^{-1}$ for AFB1, AFG1 and OTA, 0.4 and $1 \mu \mathrm{g} \mathrm{L}^{-1}$ for AFB2 and AFG2 and 64 and $160 \mu \mathrm{g} \mathrm{L}^{-1}$ for ZEA) were analysed per triplicate in three different column batches, at 58 and $62^{\circ} \mathrm{C}$, and with a mobile phase with $0.49 \%$ and $0.51 \%$ of formic acid.

In addition, the method was validated taking into account the stability of the calibration and barley samples in the chromatographic tray. A barley sample spiked to $12.5 \mu \mathrm{g} \mathrm{kg}^{-1}$ of AFB1, AFG1 and OTA, $3.1 \mu \mathrm{g} \mathrm{kg}^{-1}$ of AFB2 and AFG2 and $0.5 \mathrm{mg} \mathrm{kg}^{-1}$ of ZEA, and a calibration sample with $50 \mu \mathrm{g} \mathrm{L}^{-1}$ of AFB1, AFG1 and OTA, $12.5 \mu \mathrm{g} \mathrm{L} \mathrm{L}^{-1}$ of AFB2 and AFG2 and $2 \mathrm{mg} \mathrm{L}^{-1}$ of ZEA were analysed approximately every $75 \mathrm{~min}$, for the purpose of determining stability at $4^{\circ} \mathrm{C}$ in the chromatographic tray.

\section{Results}

\subsection{Purification conditions}

Preliminary studies on recovery were made using the IAC elution method proposed by the provider and eluting the mycotoxins with $3 \mathrm{~mL}$ of methanol. For OTA and ZEA, adequate recovery values and precision were obtained (near 100\% and RSD $<10 \%$ respectively). However, the RSD value of recovery was very high for aflatoxins (30-90\%). The influence of the volume of sample extract passed through the column $(15,20$ and $30 \mathrm{~mL})$, the evaporation temperature $\left(40,50,60\right.$ and $\left.80^{\circ} \mathrm{C}\right)$, the material used (plastic and glass tubes washed with a $\mathrm{H}_{2} \mathrm{SO}_{4}$ solution and unwashed) and the elution solvent (methanol, ethanol and acetonitrile) were studied to improve AFs recovery. 
Results suggested a possible degradation of AFs during the evaporation step of the methanol extract and an increasing loss of aflatoxins with high temperatures, while the tube material or the volume of the extract that passed through the column did not show significant changes in the recovery values (results not shown). When $3 \mathrm{~mL}$ of three pure solvents (methanol, ethanol and acetonitrile) spiked with mycotoxins were evaporated under vacuum at $40^{\circ} \mathrm{C}$, a loss of aflatoxins was found in the case of methanol, but not when they were dissolved in ethanol or acetonitrile (see table 1). To check the elution power of ethanol and acetonitrile from IAC, extracts of spiked barley samples were passed through the IAC and eluted with $3 \mathrm{~mL}$ of ethanol or acetonitrile. The best recovery value and RSD for all mycotoxins was obtained when using acetonitrile (see table 1). Therefore, acetonitrile was selected as the elution solvent of mycotoxins from the IAC, and the evaporation process was fixed at $40^{\circ} \mathrm{C}$ in a vacuum evaporator in plastic tubes.

\subsection{Chromatographic conditions}

Initially, the determination of AFs, OTA and ZEA was attempted using UHPLC-MS methodology with an ion trap detector. Although an adequate separation was obtained, the method did not satisfy the validation requirements needed so as to be considered a quantitative method, especially regarding analytical intermediate precision. Therefore, this methodology was used in the confirmation analysis, but a new method, based in UHPLC-FLD, was developed and validated as previously described in this paper.

Examples of UHPLC-FLD chromatograms obtained from a calibration and a naturally contaminated sample are shown in figure 1. Fluorescence conditions were adjusted to obtain the better analysis conditions, recording the excitation and emission spectrums while calibration standards were analysed. The wavelengths of excitation and emission 
were fixed at 365 and $440 \mathrm{~nm}$ for aflatoxins, 234 and $458 \mathrm{~nm}$ for ZEA and 225 and $469 \mathrm{~nm}$ for OTA, respectively.

\subsection{Method validation}

The addition of a known amount of mycotoxins to positive barley samples showed the increase of each mycotoxin peak without observing broadening or distortion of peak shapes. The retention time of each mycotoxin in the sample corresponded with the retention time in the calibration sample with a tolerance of $\pm 2.5 \%$. Moreover, the UHPLC-MS reanalysis of the samples confirmed the presence of mycotoxins.

The linearity study showed an adequate relation between the instrumental response (area of toxin peak) and the respective toxin concentration (x). In addition, the linearity criteria have been achieved by all of the mycotoxins in the two ranges studied (see table 2). Instrumental precision (time factor) and accuracy at the low, medium and high levels of each curve were adequate (results not shown). With regard to the instrumental precision (analyst factor), the statistical study (Mann-Whitney U test for independent samples) did not show any significant differences $(\mathrm{p}>0.05)$ among the data obtained by two operators at any of the three concentrations assayed for each toxin (results not shown).

The LOD and LOQ values for barley samples are shown in table 2. Recovery percentages at the three tested levels were between 78.2 and 109.2\%. In addition, recoveries were homogeneous at the levels assayed, which demonstrated the precision of the method (see table 3).

Robustness statistical study was developed by means of the Kruskal-Wallis test for independent samples. The study did not show any significant difference in the mycotoxin quantification as regard to the assayed values of temperature in the column 
compartment or the $\mathrm{pH}$ in the mobile phase. However, care must been taken regarding different batches of the columns (results not shown).

With regard to stability, the samples and calibration standards were stable during at least 12 hours, without observing broadening or distortion of peak shapes and with a $\mathrm{RSD}<10 \%$ of the areas of each mycotoxin peak. The results for aflatoxins coincide with those of Beaver and Rodney (1990) (Beaver \& Rodney, 1990), who observed a high stability of the aflatoxins in acidified solvents at low temperature.

\subsection{AFs, OTA and ZEA in barley samples}

This method has been successfully applied to the measurement of the mycotoxins in 20 barley samples collected during the 2007 harvest in Navarra (Spain). Of all the toxins, AFG2, AFG1 and ZEA were the least present, and none of the samples presented levels above their respective LOQ. All of the samples analysed presented levels of AFB1 above its LOD, but only $5(25 \%)$ presented quantifiable levels (> LOQ), with $0.173 \mu \mathrm{g} \mathrm{kg}^{-1}$ and $0.185 \mu \mathrm{g} \mathrm{kg}^{-1}$ being the mean of the positive values and the maximum level found, respectively. This maximum value is far below the maximum level permitted for AFB1 in cereals by the EU: $2 \mu \mathrm{g} \mathrm{kg}^{-1}$. A few of the samples presented AFB2 at very low levels; only one sample presented this toxin at a level higher than the LOQ $\left(0.042 \mu \mathrm{g} \mathrm{kg}^{-1}\right)$. In addition, 30 and $50 \%$ of the samples presented ZEA and OTA, respectively, with a level higher than the limit of detection, although the maximum level found for ZEA (1.355 $\mu \mathrm{g} \mathrm{kg}^{-1}$ ) was below its LOQ; and in the case of OTA, it was only quantifiable in one of the samples with a value of $0.157 \mu \mathrm{g} \mathrm{kg}^{-1}$, which is, as in the case of AFB1, lower than the maximum permitted level established by the EU: $5 \mu \mathrm{g} \mathrm{kg}^{-1}$. 


\section{Discussion}

During the past few years, mycotoxin analysis has focused on the simultaneous determination of several toxins with the aim of reducing the time and cost of analysis, and in addition, to find a global view of the co-occurrence of the main mycotoxins in foodstuffs. This is very important for assessing the exposure to multi-mycotoxins because it would be interesting to know the effects on animal and human health that may be caused by several toxins that are naturally present in a foodstuff.

The different chemical and physicochemical properties of the mycotoxins make it difficult to find an optimal condition or to reach to a situation of compromise that allows simultaneous extraction, purification and analysis of all of the mycotoxins. This problem has been solved in part, by using HPLC-MS because this technique does not need to derivatize the samples, and in some cases, it is possible to omit the sample pretreatment. However, this technique has some drawbacks, such as the influence of matrix on the detection or problems with quantification. For these reasons, some published HPLC-MS methods do not fulfill all of the criteria established by CEN (European Committee for Standardization) for the acceptance of an analytical method.

This paper has described the validation of a fast and simple method that explores some new chromatography advances (UHPLC and fused core columns) which allow the simultaneous analysis of six mycotoxins from three different families in a short period of time (13 minutes). The low analysis time has been due to the use of a low-volume column and a high column temperature $\left(60^{\circ} \mathrm{C}\right)$ which allow reduction of solvent viscosity and increment of the flow of the mobile phase without losing resolution. In addition, and as a result of the low analysis time, the method uses reduced solvent volumes and produces less toxic wastes. The method has a good resolution and uses the same extraction, purification and analysis procedure for all of the mycotoxins. All of 
them have been extracted from barley using a single mixture of acetonitrile-water $(60: 40, v / v)$. The extract obtained with this method is clean and can be applied to the IAC columns after dilution with PBS and centrifugation, without other cleanup processes as liquid-liquid or solid-liquid extraction.

In preliminary recovery experiments, satisfactory recovery results for ZEA and OTA were obtained using AOZ IAC column, applying the methodology indicated by the provider (methanol as elution solvent), whereas a high variation in this parameter was obtained for the four AFs. Subsequent experiments showed that the loss of AFs was produced during evaporation of the methanolic solution eluted from the columns. Problems working with AFs were reported by Beaver and Rodney (1990) who found degradation of these mycotoxins dissolved in methanol-water, acetonitrile-water and these mixtures acidified with acetic acid when left at different temperatures and under the incidence or not of light (Beaver \& Rodney, 1990). Surprisingly, and regarding methanolic elution from IAC, recovery of aflatoxins (when an extract from a barley sample is passed through the column) is better than in the case of purification of a AFs methanolic solution. These results coincide with those of Beaver and Rodney, who found that sample matrix could have some protective effect against AFs degradation.

With respect to the analysis, it is well known that AFB1 and AFG1 suffer a fluorescence quenching in aqueous solvent, therefore derivatization reaction is required to enhance their fluorescence intensity. The more frequently used methods are based on pre- or post-column derivatization. In this study, the method chosen has been the photoderivatization, which was adopted as an official AOAC method 2005.08 (Waltking \& Wilson, 2006), because of the advantages that it presents. On the one hand, the photochemical reaction allows the simultaneous determination of aflatoxins, and OTA and ZEA, while post-column iodine derivatization decrease the OTA peak and make the 
ZEA peak disappear completely; and bromine derivatization (Kobra cell) prevents the zearalenone analysis (Kok, 1994). In addition, the photo-derivatization does not require chemical reagents, pumps or other manipulations (Joshua, 1993). The photoderivatization increases AFB1 and AFG1 fluorescence intensities without producing lack of sensibility in AFB2 and AFG2, ZEA and OTA, as it can be observed in figure 2. The derivatization was made with a knitted reactor coil of $0.25 \mathrm{~mL}(5 \mathrm{~m} \times 0.25 \mathrm{~mm})$, which is smaller than that used in other research studies (Joshua, 1993, Muscarella et al., 2009), with the aim of not increasing width peak and maintaining the high resolution. The reduction of the coil size can decrease the AFB1 and AFG1 conversion rate (Joshua, 1993). However, although when the PHRED is on, the AFB1 and AFG1 fluorescence signal is lower than those found in the aforementioned works, this fact does not prevent the obtainment of good and sufficient LOD and LOQ for AFB1 and AFG1.

The method has been validated in a wide range of concentrations in accordance with the mycotoxin levels found in the literature and the maximum permitted limits by legislation. The recovery values for the six mycotoxins are adequate for their analysis and fulfill the requirements established in the Commission Regulation (EC) $\mathrm{N}^{\mathrm{o}} 401 / 2006$ (recovery between 70 and $110 \%$ in the $1-10 \mu \mathrm{g} \mathrm{kg}^{-1}$ levels, and between 50 and $120 \%$ in the $<1 \mu \mathrm{g} \mathrm{kg}^{-1}$ levels for AFs and OTA; between 60 and $120 \%$ in the $\leq 50 \mu \mathrm{g} \mathrm{kg}^{-1}$ levels, and between 70 and $120 \%$ in the $>50 \mu \mathrm{g} \mathrm{kg}^{-1}$ levels for ZEA) (European Commission, 2006b). RSD values obtained in between-day recovery experiments were between 7 and $13 \%$, which demonstrates the precision of the analytical procedure. In addition, the LOD values are below the maximum permitted limits in cereals set by legislation (European Commission, 2006a). In the case of HPLCMS methods for the analysis of cereals, the published methods usually show higher 
LODs (Beltrán, Ibáñez, Sancho \& Hernández, 2009, Frenich, Martínez, RomeroGonzález \& Aguilera-Luiz, 2009, Lattanzio, Solfrizzo, Powers \& Visconti, 2007, Spanjer, Rensen \& Scholten, 2008, Sulyok, Berthiller, Krska \& Schuhmacher, 2006, Tanaka, Takino, Sugita-Konishi \& Tanaka, 2006).

This method has been applied to the analysis of 20 barley samples. Most of the values found were < LOD or between the LOD and the LOQ values for each toxin. Quantifiable (> LOQ) levels appeared for only AFB1, AFB2 and OTA, and in few samples; the maximum levels found for these toxins were always far below the maximum levels permitted in cereals by the EU. Finally, in one sample, quantifiable levels of OTA $\left(0.157 \mu \mathrm{g} \mathrm{kg}^{-1}\right)$ and AFB1 $\left(0.177 \mu \mathrm{g} \mathrm{kg}^{-1}\right)$ co-occurred.

\section{Conclusions}

In this paper, a procedure has been validated for the quantification of six mycotoxins AFB1, AFB2, AFG1, AFG2, ZEA and OTA in barley using a UHPLC-FLD method. After applying this method to the analysis of 20 real samples, it can be concluded that said method is adequate for the purpose intented. Due to the low LODs attained, it is adequate for assuring compliance with tolerances and guidelines, for monitoring, and for carrying out survey work and research. Validation of this technique for its application in other cereal matrices such as wheat or corn is currently under investigation.

\section{Acknowledgments}

We wish to extend our gratitude to Ms. Laura Stokes for reviewing the English version of this manuscript. We thank the "Programa de Investigación Universidad de Navarra" (PIUNA) and the CAN (Caja Navarra; "Proyectos tú eliges, tú decides") for 
the financial support received. The authors are grateful to the reviewers, whose comments and suggestions have helped to improve this article.

\section{References}

Anklam, E., Stroka, J., \& Boenke, A. (2002). Acceptance of analytical methods for implementation of EU legislation with a focus on mycotoxins. Food Control, 13, 173183.

Asociación Española de farmaceúticos de la Industria (A.E.F.I) (2001). Asociación Española de Farmacéuticos de la Industria. Validación de métodos analíticos. Barcelona: AEFI.

Beaver, \& Rodney, W. (1990). Degradation of aflatoxins in common HPLC solvents. Journal of high resolution chromatography, 13(12), 833-835.

Beltrán, E., Ibáñez, M., Sancho, J. V., \& Hernández, F. (2009). Determination of mycotoxins in different food commodities by ultra-high-pressure liquid chromatography coupled to triple quadrupole mass spectrometry. Rapid Communications in Mass Spectrometry, 23(12), 1801-1809.

Bennett, J. W., \& Klich, M. (2003). Mycotoxins. Clinical Microbiology Reviews, 16(3), 497-516.

Chan, D., MacDonald, S. J., Boughtflower, V., \& Brereton, P. (2004). Simultaneous determination of aflatoxins and ochratoxin $\mathrm{A}$ in food using a fully automated immunoaffinity column clean-up and liquid chromatography- fluorescence detection. Journal of Chromatography A, 1059(1-2), 13-16. 
Cigic, I. K., \& Prosen, H. (2009). An overview of conventional and emerging analytical methods for the determination of mycotoxins. International Journal of Molecular Sciences, 10(1), 62-115.

European Commission (2006a). Commission regulation (EC) No 1881/2006 of 19 December 2006 setting maximum levels for certain contaminants in foodstuffs. Official Journal of the European Union, L 364, 5-24.

European Commission (2006b). Commission regulation (EC) No 401/2006 of 23 February 2006 laying down the methods of sampling and analysis for the official control of the levels of mycotoxins in foodstuffs. Official Journal of the European Union, L 70, 12-34.

Frenich, A. G., Martínez, J. L., Romero-González, R., \& Aguilera-Luiz, M. M. (2009). Simple and high-throughput method for the multimycotoxin analysis in cereals and related foods by ultra-high performance liquid chromatography/tandem mass spectrometry. Food Chemistry, 117(4), 705-712.

Göbel, R., \& Lusky, K. (2004). Simultaneous determination of aflatoxins, ochratoxin A, and zearalenone in grains by new immunoaffinity column. Journal of AOAC International, 87(2), 411-416.

International Agency for Research on Cancer (IARC) (2002). Some traditional herbal medicines, some mycotoxins, naphthalene and styrene. Monographs on the Evaluation of Carcinogenic Risks to Humans, 82, 21.

International Agency for Research on Cancer (IARC) (1993). Working Group on the Evaluation of Carcinogenic Risks to Humans; Some Naturally Occurring Substances: 
Food Items and Constituents, Heterocyclic Aromatic Amines and Mycotoxins. Monographs on the Evaluation of Carcinogenic Risks to Humans, 56.

Joshua, H. (1993). Determination of aflatoxins by reversed-phase high performance liquid-chromatography with postcolumn in-line photochemical derivatization and fluorescence detection. Journal of chromatography A, 654(2), 247-254.

Kok, W. T. (1994). Derivatization reactions for the determination of aflatoxins by liquid chromatography with fluorescence detection. Journal of Chromatography B: Biomedical applications, 659, 127-137.

Krska, R., Schubert-Ullrich, P., Molinelli, A., Sulyok, M., MacDonald, S., \& Crews, C. (2008). Mycotoxins analysis: An update. Food Additives and Contaminants, 25(2), 152163.

Krska, R., \& Molinelli, A. (2007). Mycotoxin analysis: state-of-the-art and future trends. Analytical and bioanalytical chemistry, 387(1), 145-148.

Kuiper-Goodman, T. (1999). Approaches to the risk analysis of mycotoxins in the food supply. Food, Nutrition and Agriculture, 23, 10-16.

Langseth, W., Ellingsen, Y., Nymoen, U., \& Okland, E. M. (1989). High-performance liquid chromatographic determination of zearalenone and ochratoxin $\mathrm{A}$ in cereals and feed. Journal of Chromatography, 478(1), 269-274.

Lattanzio, V. M. T., Solfrizzo, M., Powers, S., \& Visconti, A. (2007). Simultaneous determination of aflatoxins, ochratoxin A and Fusarium toxins in maize by liquid chromatography/tandem mass spectrometry after multitoxin immunoaffinity clean up. Rapid communications in mass spectrometry, 21, 3253-3261. 
Miraglia, M., \& Brera, C. (2002). Assessment of dietary intake of ochratoxin A by the population of EU member states. Reports on tasks for scientific cooperation, task 3.2.7, $1-153$.

Muscarella, M., Iammarino, M., Nardiello, D., Lo Magro, S., Palermo, C., Centonze, D. \& Palermo, D. (2009). Validation of a confirmatory analytical method for the determination of aflatoxins $B_{1}, B_{2}, G_{1}$ and $G_{2}$ in foods and feed materials by HPLC with on-line photochemical derivatization and fluorescence detection. Food Additives and Contaminants, 26(10), 1402-1410.

Nguyen, M., Tozovanu, M., Tran, T., \& Pfohl-Leszkowicz, A. (2007). Occurrence of aflatoxin $\mathrm{B} 1$, citrinin and ochratoxin $\mathrm{A}$ in rice in five provinces of the central region of Vietnam. Food Chemistry, 105(1), 42.

Ofitserova, M., Nerkar, S., Pickering, M., \& Torma, L. (2009). Multiresidue mycotoxin analysis in corn grain by column high-performance liquid chromatography with postcolumn photochemical and chemical derivatization: single-laboratory validation. Journal of AOAC International, 92(1), 15-25.

Pitt, J. I. (2006). Fungal ecology and the occurrence of mycotoxins. In H. Njapau, S. Trujillo, H. P. Van Egmond, \& D. L. Park, Mycotoxins and phycotoxins. Advances in determination, toxicology and exposure managementThe Netherlands: Wageningen Academic Publishers.

Spanjer, M. C., Rensen, P. M., \& Scholten, J. M. (2008). LC-MS/MS multi-method for mycotoxins after single extraction, with validation data for peanut, pistachio, wheat, maize, cornflakes, raisins and figs. Food Additives and Contaminants, 25(4), 472-489. 
Sulyok, M., Berthiller, F., Krska, R., \& Schuhmacher, R. (2006). Development and validation of a liquid chromatography/tandem mass spectrometric method for the determination of 39 mycotoxins in wheat and maize. Rapid communications in mass spectrometry, 20(18), 2649-2659.

Tanaka, H., Takino, M., Sugita-Konishi, Y., \& Tanaka, T. (2006). Development of a liquid chromatography/time-of-flight mass spectrometric method for the simultaneous determination of trichothecenes, zearalenone and aflatoxins in foodstuffs. Rapid communications in mass spectrometry, 20(9), 1422-1428.

Van Egmond, H. P., Schothorst, R. C., \& Jonker, A. M. (2007). Regulations relating to mycotoxins in food. Analytical and bioanalytical chemistry, 389(1), 147-157.

Waltking, A. E., \& Wilson, D. (2006). AOAC Official Method 2005.08. Aflatoxins in corn, raw peanuts, and peanut butter. Liquid Chromatography with Post-column Photochemical Derivatization. Journal of AOAC International, 89, 678.

Wang, Y., Chai, T., Lu, G., Quan, C., Duan, H., Yao, M., Zucker, B., \& Schlenker, G. (2008). Simultaneous detection of airborne Aflatoxin, Ochratoxin and Zearalenone in a poultry house by immunoaffinity clean-up and high-performance liquid chromatography. Environmental research, 107(2), 139.

Zöllner, P., \& Mayer-Helm, B. (2006). Trace mycotoxin analysis in complex biological and food matrices by liquid chromatography-atmospheric pressure ionisation mass spectrometry. Journal of Chromatography A, 1136(2), 123-169. 


\section{Figure captions}

Figure 1. Chromatograms obtained from a calibration sample of $0.6 \mu \mathrm{g} \mathrm{L} \mathrm{L}^{-1}$ of AFB1, AFG1 and OTA, $0.15 \mu \mathrm{g} \mathrm{L}^{-1}$ of AFB1 and AFG1 and $24 \mu \mathrm{g} \mathrm{L}^{-1}$ of ZEA ( - $)$, and a barley sample naturally contaminated (- - -).

Figure 2. Chromatograms obtained from a calibration sample $\left(4 \mu \mathrm{g} \mathrm{L}^{-1}\right.$ AFB1, AFG1 and OTA, $1 \mu \mathrm{g} \mathrm{L}^{-1}$ of AFB2 and AFG2 and $160 \mu \mathrm{g} \mathrm{L}^{-1}$ of ZEA) with UV lamp on ( - ), and UV lamp off (- - -).

\section{Figure 1}

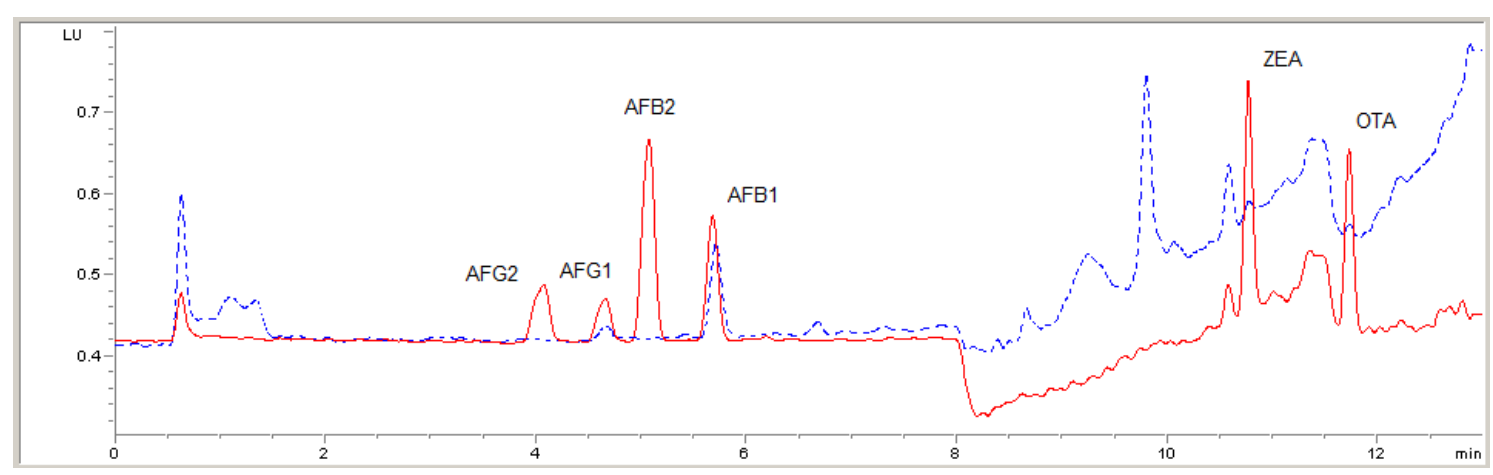




\section{Figure 2}

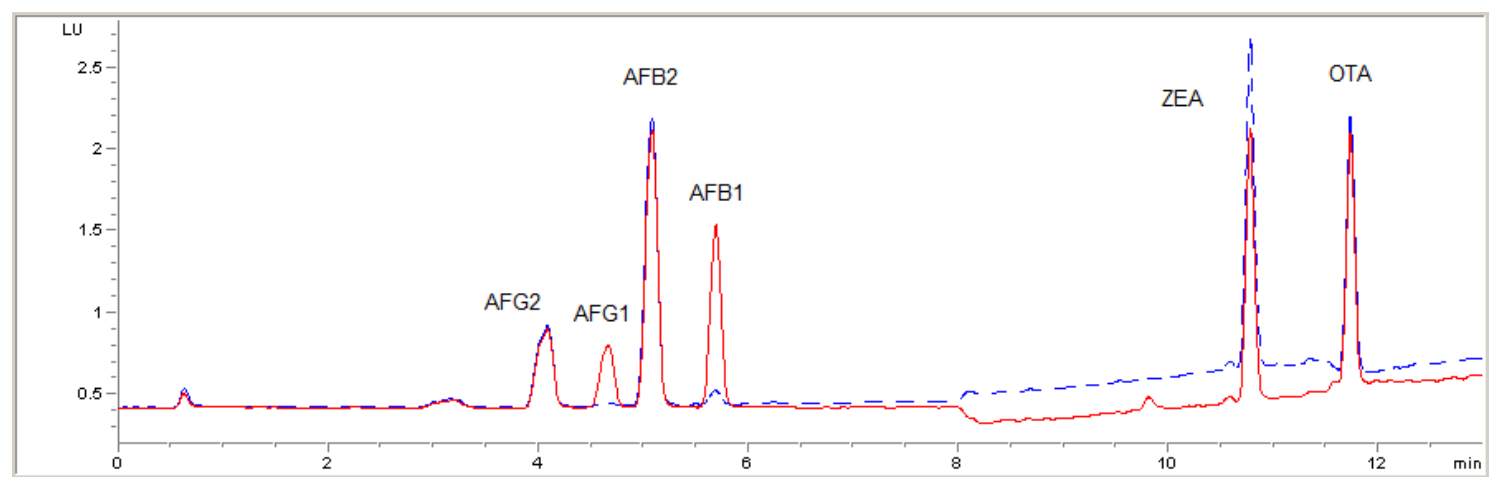

Table 1. Recovery rates of aflatoxins, ZEA and OTA using different solvents.

\begin{tabular}{ccccccc}
\hline \multirow{2}{*}{ Mycotoxin } & \multicolumn{2}{c}{$\begin{array}{c}\text { Recovery from spiked solvent }(\%) \\
(\mathrm{n}=3)(\mathrm{RSD}, \%)\end{array}$} & \multicolumn{2}{c}{$\begin{array}{c}\text { Recovery from spiked barley samples }(\%) \\
(\mathrm{n}=3)(\mathrm{RSD}, \%)\end{array}$} \\
& Methanol & Ethanol & Acetonitrile & Methanol & Ethanol & Acetonitrile \\
\hline AFG2 & $19.3(12.4)$ & $91.8(6.3)$ & $100.7(1.5)$ & $57.7(22.8)$ & $70.6(7.8)$ & $84.5(5.7)$ \\
AFG1 & $14.7(12.0)$ & $89.9(7.1)$ & $102.3(1.5)$ & $48.4(50.3)$ & $68.6(8.9)$ & $85.8(6.7)$ \\
AFB2 & $6.5(24.0)$ & $106.5(5.1)$ & $105.3(1.5)$ & $87.9(15.1)$ & $97.9(2.2)$ & $97.7(3.1)$ \\
AFB1 & $6.1(21.8)$ & $111.1(5.5)$ & $109.9(1.2)$ & $81.5(26.6)$ & $101.7(2.9)$ & $104.9(2.3)$ \\
ZEA & $101.1(10.6)$ & $97.3(4.8)$ & $94.9(4.3)$ & $116.7(0.4)$ & $93.8(1.1)$ & $94.1(1.5)$ \\
OTA & $120.8(10.7)$ & $104.4(5.2)$ & $98.1(4.6)$ & $104.9(3.0)$ & $83.3(1.8)$ & $81.3(3.8)$ \\
\hline
\end{tabular}


Table 2. Limit of detection (LOD), limit of quantification (LOQ) and linearity data.

\begin{tabular}{|c|c|c|c|c|c|c|c|}
\hline Mycotoxin & $\underset{\left(\mathrm{ng} \mathrm{kg}^{-1}\right)}{\mathrm{LOD}}$ & $\underset{\left(\mathrm{ng} \mathrm{kg}^{-1}\right)}{\mathrm{LOQ}}$ & Range & curve equation & $r^{2}$ & $\begin{array}{l}\text { Slope confident } \\
\text { interval } \\
(p=95 \%)\end{array}$ & $\begin{array}{c}y \text {-intercep } \\
\text { confident interval } \\
(p=95 \%)\end{array}$ \\
\hline \multirow{2}{*}{ AFG2 } & \multirow{2}{*}{3.0} & \multirow{2}{*}{37.5} & $0.15-1 \mu \mathrm{g} \mathrm{L}^{-1}$ curve & $y=4.87 x+0.04$ & 0.997 & $4.52,5.22$ & $-0.17,0.25$ \\
\hline & & & $1-10 \mu \mathrm{g} \mathrm{L}^{-1}$ curve & $y=5.36 x-0.69$ & 0.999 & $5.12,5.61$ & $-2.16,0.77$ \\
\hline \multirow{2}{*}{ AFG1 } & \multirow{2}{*}{15.0} & \multirow{2}{*}{150.0} & $0.6-4 \mu \mathrm{g} \mathrm{L}{ }^{-1}$ curve & $y=0.79 x+0.05$ & 0.996 & $0.72,0.86$ & $-0.11,0.22$ \\
\hline & & & $4-40 \mu \mathrm{g} \mathrm{L}^{-1}$ curve & $y=0.96 x-0.81$ & 0.999 & $0.92,1.00$ & $-1.77,0.15$ \\
\hline \multirow{2}{*}{ AFB2 } & \multirow{2}{*}{0.5} & \multirow{2}{*}{37.5} & $0.15-1 \mu \mathrm{g} \mathrm{L}^{-1}$ curve & $y=13.62 x-0.11$ & 0.998 & $12.78,14.46$ & $-0.62,0.40$ \\
\hline & & & $1-10 \mu \mathrm{g} \mathrm{L}^{-1}$ curve & $y=14.18 x-1.64$ & 0.999 & $13.55,14.82$ & $-5.50,2.22$ \\
\hline \multirow{2}{*}{ AFB1 } & \multirow{2}{*}{7.0} & \multirow{2}{*}{150.0} & $0.6-4 \mu \mathrm{g} \mathrm{L} \mathrm{L}^{-1}$ curve & $y=1.92 x-0.11$ & 0.998 & $1.79,2.05$ & $-0.42,0.22$ \\
\hline & & & $4-40 \mu \mathrm{g} \mathrm{L}^{-1}$ curve & $\mathrm{y}=2.07 \mathrm{x}-1.34$ & 0.999 & $1.98,2.16$ & $-3.54,0.86$ \\
\hline \multirow{2}{*}{ ZEA } & \multirow{2}{*}{340.0} & \multirow{2}{*}{6000.0} & $24-160 \mu \mathrm{g} \mathrm{L}{ }^{-1}$ curve & $y=0.06 x-0.13$ & 0.997 & $0.05,0.06$ & $-0.56,0.30$ \\
\hline & & & $160-1600 \mu \mathrm{g} \mathrm{L}^{-1}$ curve & $y=0.06 x-1.73$ & 0.999 & $0.06,0.06$ & $-4.52,1.07$ \\
\hline \multirow{2}{*}{ OTA } & \multirow{2}{*}{13.0} & \multirow{2}{*}{150.0} & 0.6 - $4 \mu \mathrm{g} \mathrm{L}{ }^{-1}$ curve & $\mathrm{y}=2.19 \mathrm{x}-0.23$ & 0.998 & $2.07,2.32$ & $-0.54,0.08$ \\
\hline & & & $4-40 \mu \mathrm{g} \mathrm{L}^{-1}$ curve & $\mathrm{y}=2.18 \mathrm{x}-0.90$ & 0.999 & $2.07,2.29$ & $-3.53,1.72$ \\
\hline
\end{tabular}


Table 3. Within and between-day precision and recovery.

\begin{tabular}{|c|c|c|c|c|c|}
\hline \multirow{2}{*}{ Mycotoxin } & \multirow{2}{*}{$\begin{array}{l}\text { Toxin added } \\
\quad\left(\mu \mathrm{g} \mathrm{kg}^{-1}\right)\end{array}$} & \multicolumn{2}{|c|}{ Within-day recovery (RSD; \%) } & \multicolumn{2}{|c|}{ Between-day recovery (RSD; \%) } \\
\hline & & $(\mathrm{n}=3)$ & Global $(\mathrm{n}=9)$ & $(\mathrm{n}=9)$ & $\begin{array}{c}\text { Global } \\
(\mathrm{n}=27)\end{array}$ \\
\hline \multirow{3}{*}{ AFG2 } & 0.0375 & $85.9(5.5)$ & \multirow{3}{*}{$80.3(10.4)$} & $77.7(9.5)$ & \multirow{3}{*}{$78.2(10.6)$} \\
\hline & 0.25 & $83.2(9.9)$ & & $80.3(12.6)$ & \\
\hline & 2.5 & $71.7(6.4)$ & & $76.5(6.4)$ & \\
\hline \multirow{3}{*}{ AFG1 } & 0.15 & $79.7(1.4)$ & \multirow{3}{*}{$80.3(7.7)$} & $86.7(8.4)$ & \multirow{3}{*}{$85.1(12.1)$} \\
\hline & 1 & $84.3(11.1)$ & & $86.3(15.9)$ & \\
\hline & 10 & $77.0(6.4)$ & & $82.3(8.6)$ & \\
\hline \multirow{3}{*}{ AFB2 } & 0.0375 & $91.6(3.0)$ & \multirow{3}{*}{$93.3(3.0)$} & $99.2(6.3)$ & \multirow{3}{*}{$97.0(7.5)$} \\
\hline & 0.25 & $95.7(2.2)$ & & $99.6(8.5)$ & \\
\hline & 2.5 & $92.6(2.5)$ & & $92.1(2.7)$ & \\
\hline \multirow{3}{*}{ AFB1 } & 0.15 & $97.2(5.7)$ & \multirow{3}{*}{$93.4(5.0)$} & $97.3(4.2)$ & \multirow{3}{*}{$94.2(7.3)$} \\
\hline & 1 & $93.2(2.1)$ & & $96.9(7.9)$ & \\
\hline & 10 & $89.9(3.7)$ & & $88.4(3.6)$ & \\
\hline \multirow{3}{*}{ ZEA } & 6 & $104.4(2.7)$ & \multirow{3}{*}{$104.3(5.5)$} & $115.1(9.8)$ & \multirow{3}{*}{$109.2(10.9)$} \\
\hline & 40 & $109.3(5.5)$ & & $115.5(6.6)$ & \\
\hline & 400 & $99.0(2.6)$ & & $97.0(2.1)$ & \\
\hline \multirow{3}{*}{ OTA } & 0.15 & $83.3(11.5)$ & \multirow{3}{*}{$81.5(7.8)$} & $89.3(9.2)$ & \multirow{3}{*}{$83.2(10.5)$} \\
\hline & 1 & $79.9(6.4)$ & & $83.6(5.4)$ & \\
\hline & 10 & $81.2(7.4)$ & & $76.7(9.7)$ & \\
\hline
\end{tabular}

